\title{
Keratolytic Winter Erythema: An Update
}

\author{
Michèle Ramsay ${ }^{a} \quad$ Thandiswa Ngcungcu ${ }^{a}$ Wayne Grayson ${ }^{b, c}$ \\ a'Division of Human Genetics, School of Pathology and Sydney Brenner Institute for \\ Molecular Bioscience, Faculty of Health Sciences, University of the Witwatersrand, \\ Johannesburg, South Africa; ${ }^{b}$ Division of Anatomical Pathology, School of Pathology, \\ Faculty of Health Sciences, University of the Witwatersrand, Johannesburg, South Africa; \\ 'Ampath National Laboratories, Johannesburg, South Africa
}

\section{Keywords}

Keratolytic winter erythema $\cdot$ Skin $\cdot$ Oudtshoorn disease $\cdot$ Keratoderma $\cdot$ Erythrokeratolysis hiemalis

\begin{abstract}
Keratolytic winter erythema (KWE) is a rare autosomal dominant keratoderma affecting primarily the palms and soles, manifesting with recurrent waves of erythema followed by epidermal peeling. The condition is so named in view of its anecdotal worsening during the winter months. It is highly penetrant but shows considerable individual clinical variability, waning and reappearing throughout the life course. Histologically, early established lesions of KWE manifest with degenerative changes involving the Malpighian layer, with associated absence of the stratum granulosum. The damaged zone undergoes parakeratotic transformation and subsequent centrifugal ejection. Thick peeling occurs when the stratum corneum eventually separates off as a result of a keratolytic split occurring above, through or below the parakeratotic zone. Reconstitution of the stratum granulosum ensues. KWE is caused by a duplication of an intergenic enhancer element upstream of the cathepsin B gene on chromosome 8 . This leads to the upregulation of cathepsin $B$ in the stratum granulosum and subsequent peeling of the epidermis as the end result. With elucidation of the molecular pathology of KWE, new therapeutic approaches to KWE may be considered.


Ramsay et al.: Keratolytic Winter Erythema

\section{Introduction}

Keratolytic winter erythema (KWE; OMIM \#148370) is an orphan disease rarely seen and not always diagnosed. As an autosomal dominant trait, a family history is an important starting point where one of the parents is expected to be affected, though the level of clinical involvement may vary from person to person, even within a single family. A curious feature is the element of exacerbation ascribed to specific environmental and physiological triggers. These remain poorly understood and largely anecdotal, for example, that it is worse in the winter months - hence its name.

Due to a founder effect, KWE is more common in South Africa among White and Coloured (or admixed) communities [1-3]. It has been successfully traced back to a single founder, the mariner François Renier Duminy (born 1747 in France), who later in life settled in the Cape of Good Hope, leaving many descendants with KWE now living in South Africa [4]. Curiously, few cases of KWE are documented in his native country, France.

It took about 20 years to identify the genetic cause of KWE, counting from the time of the linkage study that examined the segregation of genetic markers in large families and first pinpointed the location, to the time of finding the actual genetic mutation. The search for the mutation started from the localisation of the genetic position of the KWE trait in 1997 to chromosome 8p23.1-p22 [5] and was followed by several studies examining the coding regions of good biological candidate genes in the region [6, 7]. It was only in 2017 that the genetic causal mutation was discovered [8]. The mutation was not in a gene or coding sequence, but an intergenic region upstream of cathepsin B (CTSB). It is a segmental duplication that includes an enhancer element that is active in keratinocyte development $[8,9]$. There are two different duplications, one found in South African KWE families (a 7.67-kb duplication) and the other in Norwegian families (a 15.93-kb duplication). These duplications overlap in the region of the enhancer [8], and functional studies show that the enhancer is specific to the regulation of $C T S B$.

In the presence of the duplication, CTSB gene expression is elevated in the stratum granulosum of affected individuals. We therefore concluded that the duplication of the noncoding enhancer element is the cause of the upregulation of cathepsin B and is likely modulated by specific environmental and physiological triggers.

\section{Details and Discussion}

KWE has been an enigma for many decades and still presents significant treatment challenges. Although we have had good clinical and histopathological descriptions for some time and have now progressed to an understanding of the genetic basis and its cellular consequences, we remain curious about the triggers that lead to exacerbation of the clinical phenotype. Below are a detailed clinical description and histopathological observations of KWE, with previously unpublished and high-resolution photographs to assist others in making a diagnosis of KWE.

\section{Clinical Description}

KWE characteristically presents with erythematous, peeling skin and blistering of the palmoplantar skin of the extremities. A slowly migratory, gyrate, annular erythema may also be encountered on the extremities $[1,2,8]$. The condition usually manifests in the first 5 years of life, with the most severely affected individuals already showing signs of the condition during infancy. Although not definitive, KWE appears to have a propensity for worsening during winter in some geographic locations, while improvement or disappearance 
Fig. 1. Keratolytic winter erythema, with characteristic blistering and peeling of the palms.
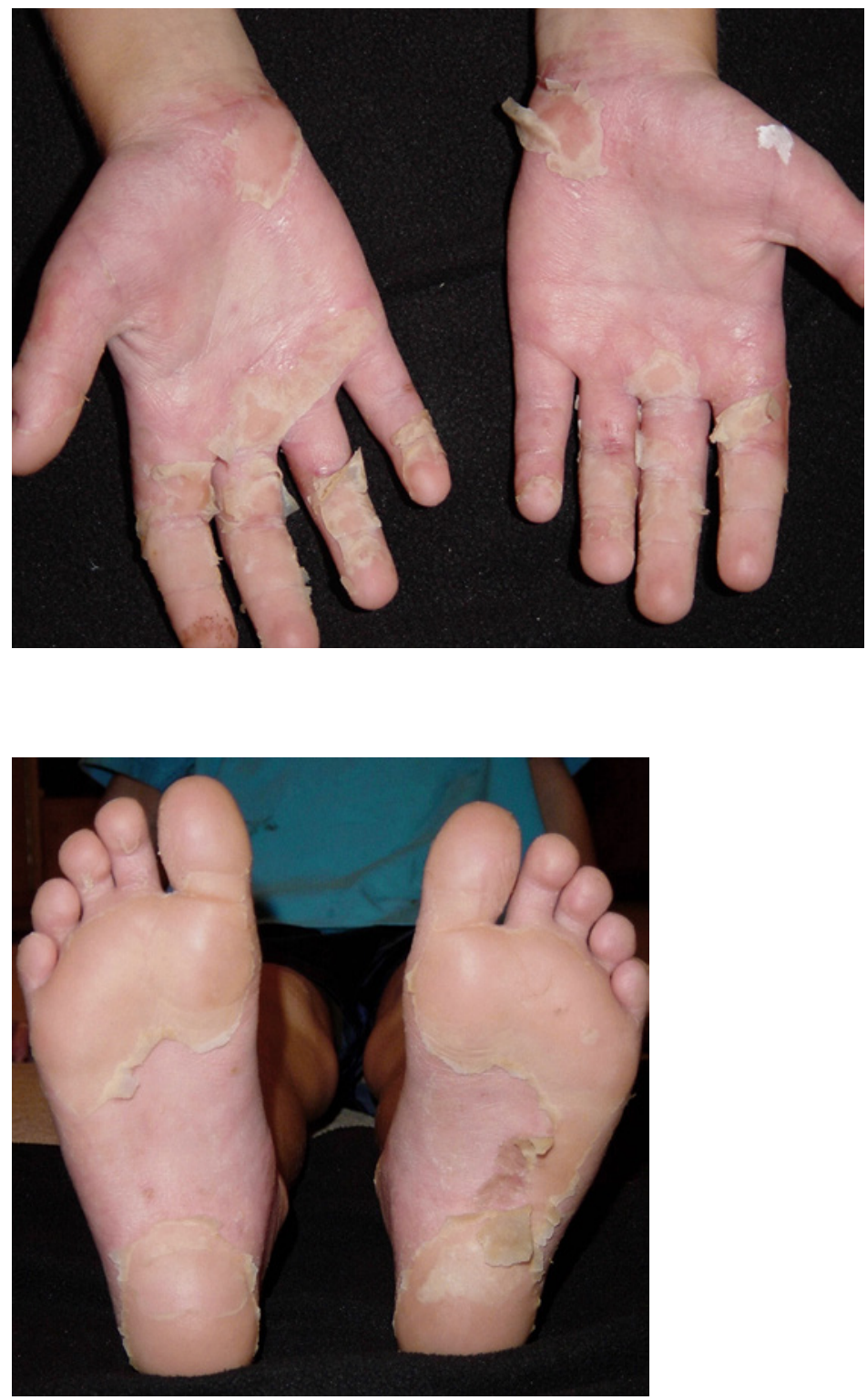

Fig. 2. Plantar involvement in keratolytic winter erythema. Note the propensity for the peeling to become arrested at the skin creases.

of lesions may occur during the summer months. Recurring cycles of palmar and plantar erythema are followed by hyperkeratosis and the formation of dry, superficial blisters with eventual centrifugal peeling (Fig. 1). The peeling is multifocal but tends to become arrested at the skin creases (Fig. 2). The recrudescent cycles usually last between 6 and 8 weeks. The most severe cases have a history of onset in infancy, show year-round persistence of lesions and/or extension of the disease process onto the dorsal aspects of the hands and feet. Involvement of the lower legs and knees is sometimes seen. Buttock or thigh lesions, however, are relatively uncommon [1,2]. Most patients experience only mild discomfort, although associated palmoplantar sweating and a pungent odour are frequently encountered $[1,8]$. Known triggers include cold weather, febrile illness and physical or emotional stress [8]. There is often an improvement in the condition during pregnancy and beyond the age of 30 years [1]. 


\section{Dermato pathology}

Fig. 3. Medium-power view illustrating the typical histological features of an active lesion, including degenerative cellular changes in the epidermis, absence of the granular cell layer, and adjacent parakeratosis. HE. Original magnification, $\times 200$.

Fig. 4. High-power view of an early lesion. Note the degenerative epidermal alterations, with cellular pallor, nuclear pyknosis, release of eosinophilic granular material and early adjacent parakeratosis. HE. Original magnification, $\times 400$.

Fig. 5. Medium-power view highlighting the degeneration, spongiosis and compact parallel orientation of damaged cells in the upper epidermis on the right. The latter is said to resemble incipient parakeratosis. HE. Original magnification, $\times 200$.

\begin{tabular}{l|l}
\hline Dermatopathology 2019;6:126-132 \\
\hline DOI: 10.1159/000496338 & $\begin{array}{l}\text { @ 2019 The Author(s). Published by S. Karger AG, Basel } \\
\text { www.karger.com/dpa }\end{array}$ \\
\hline
\end{tabular}
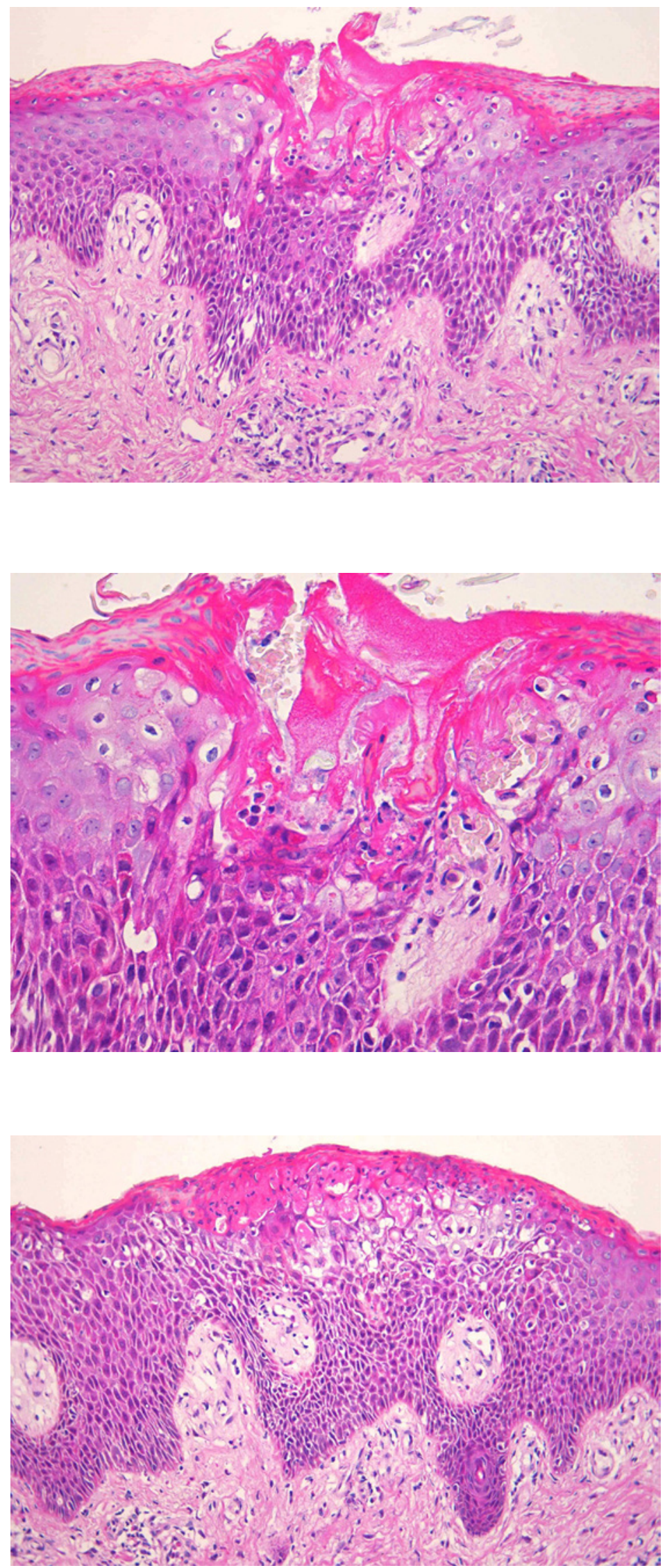
Fig. 6. High-power view of a late lesion. Note the ejected, parakeratotically transformed cells, which are now sandwiched below the old stratum corneum. There is early reconstitution of the stratum granulosum on the righthand side of the field. HE. Original magnification, $\times 400$.

Ramsay et al.: Keratolytic Winter Erythema

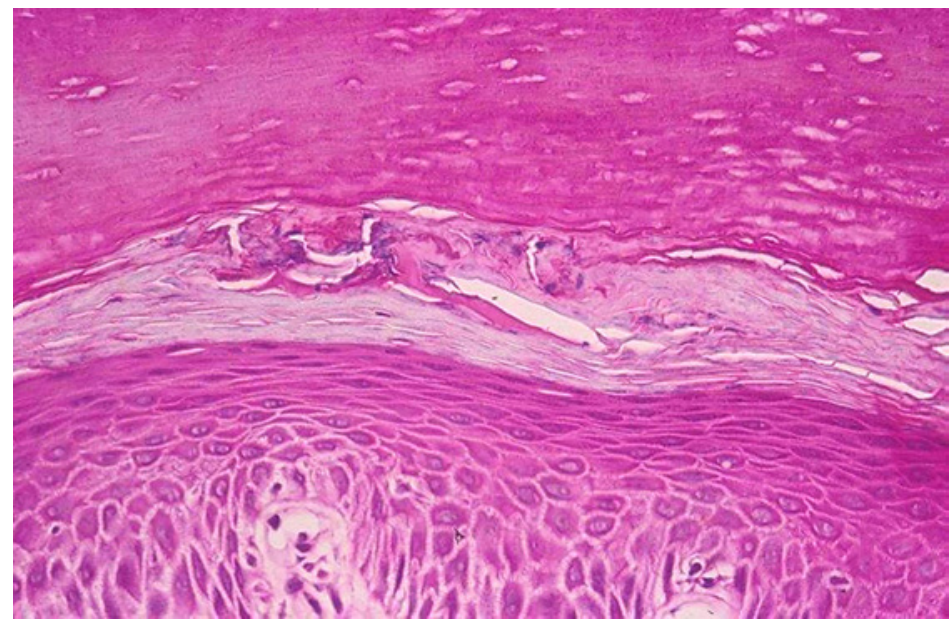

Fig. 7. Low-power view of an established peeling lesion. The plane of cleavage is clearly visible on the right-hand side of this field. Note how the parakeratotic layer is hoisted up and sandwiched between the old stratum corneum above and the new stratum corneum below. HE. Original magnification, $\times 100$.

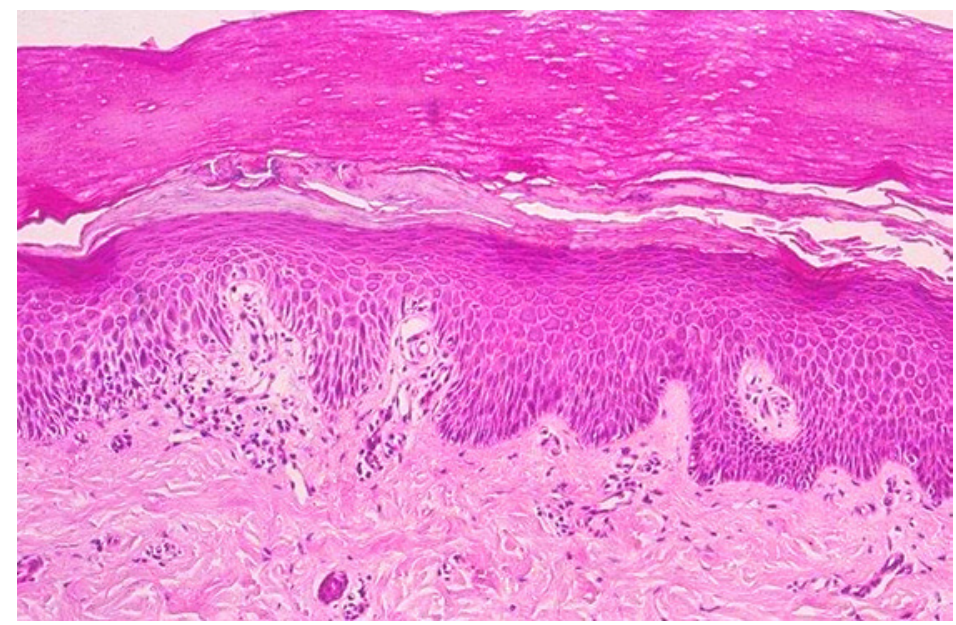

\section{Histopathology}

Only a limited number of descriptions of the histomorphology of KWE lesions exist in the English-language medical literature [1,2]. According to the landmark historical text by Findlay and Morrison [2], the outstanding histological feature of this condition is "necrobiosis of the Malpighian layer," with associated absence of an overlying stratum granulosum in early, established lesions (Fig. 3). In general, there are numerous scattered vacuolated keratinocytes throughout the epidermis with formation of a broad band in the upper epidermis showing balloon degeneration. The keratinocytes in the mid- and lower epidermis have small pyknotic nuclei and relatively scant cytoplasm, while in the upper layers the cells are larger, with abundant cytoplasm. It is within these layers that prominent ballooning degeneration is seen. Subsequent degeneration results in the damaged cells becoming compacted in a parallel manner, with a granular eosinophilic band and overlying parakeratosis. Degeneration of entire groups of cells is associated with the release of eosinophilic granular debris (Fig. 4). A combination of these degenerative changes and mild spongiosis may also result in the formation of a primordial vesicle (Fig. 5). The damaged zone becomes parakeratotic and is ploughed out centrifugally by the recovering layers behind it. In essence, it becomes sandwiched between the old stratum corneum above and the new stratum corneum below (Fig. 6). 
The epidermis seals up the centrifugal zonal damage to the stratum spinosum, with the continuous compact parakeratotic lamina ejected from behind. Thick peeling ensues when the stratum corneum eventually separates off in its plane, with the keratolytic split occurring above, through or below the parakeratotic zone (Fig. 7). There is thickening of the stratum granulosum in the wake of this process. Conspicuous regenerative basal cell hyperplasia (historically referred to as "basiliosis") occurs adjacent to the parakeratotic zone [1,2]. The process is cyclical, with rapid restoration of the normal epidermal structure. There is a minimal inflammatory reaction, with only occasional lymphocytes observed in the superficial dermis.

\section{Advances in Genetics and Pathogenesis}

The discovery of the genetic basis of the KWE phenotype and the functional studies supporting dysregulation of cathepsin B, specifically in the stratum granulosum, are providing a starting point to a deeper understanding of the clinical phenotype [8]. The intermittent and variable nature of the appearance of the palmoplantar lesions suggests that there are specific triggering events. These remain unclear and will require further longitudinal investigations focusing on the initiation and progression of the clinical features. The current advances could, however, lead to the development of treatments aimed at suppressing or downregulating cathepsin B in the skin of individuals with active KWE.

\section{Acknowledgements}

This paper is dedicated in loving memory to the late Prof. Torunn Friskerstrand of the Center for Medical Genetics and Molecular Medicine, Haukeland University Hospital, Bergen, Norway. Dr. Sian Hartshorne, formerly of the Division of Dermatology, Faculty of Health Sciences, University of the Witwatersrand, Johannesburg, South Africa, is thanked for providing Figures 1 and 2. The authors are indebted to Prof. Peter Hull, Division of Clinical Dermatology and Cutaneous Science, Dalhousie University, Halifax, NS, Canada for his critical appraisal of the manuscript.

\section{Statement of Ethics}

The authors have no ethical conflicts to disclose for this review.

\section{Disclosure Statement}

The authors have no conflicts of interest to declare.

\section{Author Contributions}

T.N. and M.R. are responsible for the abstract, introduction, and overview of the molecular genetics and pathogenesis sections. W.G. is responsible for the sections on clinical features and histopathology, as well as for photomicrography and the general formatting of the manuscript. 
Ramsay et al.: Keratolytic Winter Erythema

\section{References}

1 Findlay GH, Nurse GT, Heyl T, Hull PR, Jenkins T, Klevansky H, et al. Keratolytic winter erythema or "Oudtshoorn skin": a newly recognized inherited dermatosis prevalent in South Africa. S Afr Med J. 1977 Nov;52(22): 871-4.

2 Findlay GH, Morrison JG. Erythrokeratolysis hiemalis - keratolytic winter erythema or "Oudtshoorn Skin". A new epidermal genodermatosis with its histological features. Br J Dermatol. 1978 May;98(5):491-5.

3 Hull PR, Hobbs A, Aron S, Ramsay M. The elusive gene for keratolytic winter erythema. S Afr Med J. 2013 Oct; 103(12 Suppl 1):961-5.

4 Hull P. Keratolytic winter erythema (Oudtshoorn disease): clinical, genetic and ultraspectural aspects [PhD thesis]. Johannesburg: University of the Witwatersrand; 1986.

5 Starfield M, Hennies HC, Jung M, Jenkins T, Wienker T, Hull P, et al. Localization of the gene causing keratolytic winter erythema to chromosome 8p22-p23, and evidence for a founder effect in South African Afrikaansspeakers. Am J Hum Genet. 1997 Aug;61(2):370-8.

6 Appel S, Filter M, Reis A, Hennies HC, Bergheim A, Ogilvie E, et al. Physical and transcriptional map of the critical region for keratolytic winter erythema (KWE) on chromosome 8p22-p23 between D8S550 and D8S1759. Eur J Hum Genet. 2002 Jan;10(1):17-25.

7 Hobbs A, Aron S, Hartshorne S, Hull PR, Ramsay M. Exclusion of CTSB and FDFT1 as positional and functional candidate genes for keratolytic winter erythema (KWE). J Dermatol Sci. 2012 Jan;65(1):58-62.

8 Ngcungcu T, Oti M, Sitek JC, Haukanes BI, Linghu B, Bruccoleri R, et al. Duplicated enhancer region increases expression of CTSB and segregates with keratolytic winter erythema in South African and Norwegian families. Am J Hum Genet. 2017 May;100(5):737-50.

9 Oti M, Falck J, Huynen MA, Zhou H. CTCF-mediated chromatin loops enclose inducible gene regulatory domains. BMC Genomics. 2016 Mar;17(1):252. 